\title{
Estudo do sabão de óleo de mamona (Ricinus communis) como inibidor de corrosão em fluido salino
}

\author{
Study of castor oil soap (Ricinus communis) as a corrosion inhibitor in saline fluid
T. S. Santana ${ }^{1 *}$; E. R. N. Lima ${ }^{1 *}$; G. F. Silva ${ }^{1}$; M. S. Silva ${ }^{1}$; A. O. Wanderley $\mathrm{Neto}^{2}$ \\ ${ }^{1}$ Programa de Pós-Graduação em Engenharia Química, Universidade Federal de Sergipe, 49100-000, São Cristóvão- \\ Sergipe, Brasil. \\ ${ }_{2}^{2}$ Departamento de Química, Universidade Federal do Rio Grande do Norte, 59078-970, Natal-Rio Grande do Norte, \\ Brasil
}

*tam.santana@hotmail.com

(Recebido em 23 de julho de 2017; aceito em 07 de maio de 2018)

\begin{abstract}
A corrosão é um processo de deterioração de material metálico, ocorrendo mais comumente em meios aquosos em presença de eletrólitos, à exemplo da água produzida juntamente com petróleo que em contato com a superfície dos oleodutos geram corrosão. De forma a evitar que esse processo ocorra, inibidores de corrosão podem ser utilizados, aumentando a vida útil dos oleodutos. No presente trabalho foi obtido um tensoativo a partir de óleo vegetal, o óleo da mamona saponificado (OMS), que foi avaliado quanto à capacidade de inibição da corrosão frente ao aço carbono. O óleo de mamona foi saponificado a partir do valor calculado do índice de saponificação (IS) e caracterizado pelo índice de acidez (IA). A eficiência de inibição à corrosão foi avaliada pela técnica de voltametria de varredura linear, pelo método de resistência à polarização onde é possível avaliar a eficiência de um composto frente à corrosão gerada pela intensidade de corrente das reações de corrosão. Dessa forma, o OMS foi obtido e avaliado como inibidor de corrosão do aço carbono em fluido salino contendo $\mathrm{NaCl} 3,5 \%$. O resultado obtido para o índice de acidez foi de 0,119 , garantindo assim a qualidade do óleo, o índice de saponificação obtido foi de 161,73 g KOH/g, um pouco abaixo da literatura porém satisfatório. A polarização linear comprovou ação inibitória do óleo de mamona saponificado em meio salino uma vez que ocorreu decréscimo da corrente de corrosão e deslocamento do potencial de corrosão para valores mais positivos, com eficiência máxima de 89,6\%.

Palavras-chave: Óleo de mamona, corrosão, fluido salino, sustentabilidade, tensoativo.
\end{abstract}

Corrosion is a process of deterioration of metallic material, occurring most commonly in aqueous media in the presence of electrolytes, such as produced water along with oil that in contact with the surface of the pipelines generate corrosion. To prevent this process from occurring, corrosion inhibitors can be used, increasing the life of pipelines. In the present work, a surfactant was obtained from vegetable oil, the saponified castor oil (OMS), which was evaluated for its ability to inhibit corrosion against carbon steel. Castor oil was saponified from the calculated value of the saponification index (SI) and characterized by the acid number (IA). The corrosion inhibition efficiency was evaluated by the linear scanning voltammetry technique by the polarization resistance method where it is possible to evaluate the efficiency of a compound against the corrosion generated by the current intensity of the corrosion reactions. Thus, the who was obtained and evaluated as a corrosion inhibitor of carbon steel in saline containing $\mathrm{NaCl} 3,5 \%$. The result obtained for the acidity index was of 0,119 thus guaranteeing the quality of the oil, the saponification index obtained was of $161,73 \mathrm{mg} \mathrm{KOH} / \mathrm{g}$ oil a little bit under the literaute but still considered satisfactory.The linear polarization showed an inhibitory action of the saponified castor oil in saline medium, since the corrosion current decreased and the corrosion potential displaced to more positive values, with a maximum efficiency of $89,6 \%$. Keywords: Castor oil, corrosion, saline fluid, sustainability, surfactant

\section{INTRODUÇÃO}

O petróleo é um produto natural originado a partir da deposição e decomposição de matéria orgânica, que se apresenta como líquido oleoso, inflamável, menos denso que a água, com cheiro característico e coloração entre o negro e castanho claro [1]. É explorado de forma a se obter diferentes compostos à partir da sua destilação fracionada. 
Em diversos reservatórios, devido a presença de aquíferos próximos a zona produtora ou relacionado à água injetada para aumento da recuperação do óleo, há a extração de água junto ao petróleo, sendo essa água denominada água produzida (AP). A quantidade de AP associada ao óleo pode variar ao longo da vida produtiva do poço, podendo alcançar valores de 50 - $100 \%$ em campos denominados maduros [1, 2]. A água produzida geralmente apresenta alta salinidade, com partículas de óleo em suspensão. Dentre os minerais encontra-se os sólidos dissolvidos totais (SDT) constituintes inorgânicos compostos por cátions $\left(\mathrm{Na}^{+}, \mathrm{K}^{+}, \mathrm{Ca}^{2+}, \mathrm{Mg}^{2+}, \mathrm{Ba}^{2+}, \mathrm{Sr}^{2+}, \mathrm{Fe}^{2+}\right)$ e ânions $\left(\mathrm{Cl}^{-}, \mathrm{SO}_{4}{ }^{2-}, \mathrm{CO}_{3}{ }^{2-}, \mathrm{HCO}_{3}{ }^{-}\right)$onde predominam o $\mathrm{Na}^{+}$e o $\mathrm{Cl}^{-}$. A concentração de STD na AP pode variar de valores menores que $1000 \mathrm{mg} \mathrm{L}^{-1}$ (próximo a água potável $250 \mathrm{mg} \mathrm{L}^{-1}$ ) até $300.000 \mathrm{mg} \mathrm{L}^{-}$ 1 (maior que a da água do mar $35000 \mathrm{mg} \mathrm{L}^{-1}$ ), dependendo da localização geográfica e da idade e tipo do reservatório de petróleo [3].

O transporte do óleo "onshore" no Brasil é feito principalmente via oleodutos, constituído em grande parte de aço carbono, uma liga ferrosa relativamente complexa, que contém em torno de $0,008 \%$ a 2,0 \% em massa de carbono. Por possuir baixo custo e apresentar boa resistência mecânica, o aço carbono é um material bastante atrativo para construção de estruturas e equipamentos, sendo amplamente utilizado na indústria [4]. Porém, sem adição de elementos de liga, o aço carbono fica mais suscetível à corrosão, uma vez que esse é difícil de passivar, pois seu constituinte principal é o ferro, metal próximo à extremidade anódica da série galvânica, ocorrendo a formação de filmes de óxidos/hidróxidos ou sais pouco aderentes e com mínima capacidade de proteger a superfície contra o ataque dos meios corrosivos $[4,5]$.

A corrosão é um fenômeno que ocorre de modo espontâneo gerando a deterioração de um material geralmente metálico por ação química ou eletroquímica diminuindo a durabilidade e o desempenho desses materiais [6]. Segundo Wolynec (2003) [7], a corrosão ocorre em 90\% dos casos em meios aquosos, conhecida como corrosão eletroquímica, devido à existência de uma diferença de potencial entre os meios, gerando um fluxo de corrente elétrica e transferência de elétrons que se difundem através da superfície do metal sendo transferidos da região anódica (onde ocorre a oxidação) para a catódica (onde ocorre a redução). A corrosão gera prejuízos de alto custo na indústria, devido ao desperdício considerável de investimento, acidentes de trabalho e até perda de vidas humanas por conta de falta de segurança nos equipamentos. A substituição de equipamentos deteriorados pela corrosão é bastante onerosa, principalmente quando comparados aos custos indiretos associados à prevenção de danos, como uso de técnicas anticorrosivas, resultando na determinação de uso de aditivos químicos para o retardamento ou inibição do processo de corrosão [8].

A corrosão ocorre via reações de oxi-redução (ou redox), onde há simultaneamente a perda e ganho de elétrons por espécies químicas presentes num mesmo meio em presença de eletrólitos, com transferência de elétrons de uma espécie redutora para outra oxidante.

No aço carbono, como o ferro é o principal componente, as mudanças devido a corrosão ocorrem na superfície do metal, de acordo com as reações químicas demonstradas nas equações de (1) à (4) a seguir:

$$
\begin{aligned}
\mathrm{Fe} \rightarrow & \mathrm{Fe}^{2+}+2 e^{-} \\
& \mathrm{O}_{2}+2 \mathrm{H}_{2} \mathrm{O}+4 e^{-} \rightarrow 4 \mathrm{OH}^{-}
\end{aligned}
$$

As reações (1) e (2) ocorrem simultaneamente nos processos corrosivos, sendo a combinação de ambas a descrição da reação de corrosão resultando na formação do $2 \mathrm{Fe}(\mathrm{OH})_{2}$, conforme abaixo:

$$
\begin{gathered}
2 \mathrm{Fe}+\mathrm{O}_{2}+2 \mathrm{H}_{2} \mathrm{O} \rightarrow 2 \mathrm{Fe}(\mathrm{OH})_{2} \\
\mathrm{Fe}(\mathrm{OH})_{2}+\mathrm{O}_{2} \rightarrow \mathrm{H}_{2} \mathrm{O}+2 \mathrm{Fe}_{2} \mathrm{O}_{3} \cdot \mathrm{H}_{2} \mathrm{O}
\end{gathered}
$$

De forma a evitar processos corrosivos em oleodutos, pode-se realizar injeção de produtos químicos, chamados inibidores de corrosão. Eles podem ser classificados quanto à composição (orgânicos e inorgânicos) e com relação ao comportamento químico, classificação mais usual encontrada na literatura e descrita por [6], dividida em anódicos, catódicos e de adsorção. 
Segundo Wolynec (2003) [7] os inibidores anódicos são aqueles que atuam reprimindo as reações anódicas. Geralmente reagem com o produto de corrosão já formado, ocasionando um filme aderente e insolúvel na superfície do metal, causando a polarização anódica. Já os inibidores de corrosão catódicos atuam reduzindo a velocidade das reações catódicas, precipitando na superfície do metal, deixando-a mais polarizada negativamente e assim aumentando a resistência a corrosão, pois diminui a difusão do oxigênio no meio proporcionando um decréscimo na velocidade das reações de redução [9]. Para os inibidores de adsorção a atuação é por conta da formação de películas protetoras tanto nas regiões catódicas como nas anódicas, capazes de revestir toda superfície do metal, minimizando e até impedindo as reações de dissolução do metal pois interfere na ação eletroquímica. As películas de proteção geradas por esses inibidores podem ser afetadas por diversos fatores como a velocidade do fluido, concentração do inibidor, temperatura do sistema, tempo de contato entre o inibidor e a superfície do metal e a composição do fluido no sistema [4].

Inibidores de corrosão a base de extratos de plantas ricas em alcaloides ou substâncias fenólicas também agem como bons indicadores e agregam valor ao produto final uma vez que possuem o apelo sustentável, como alternativa ao uso de substâncias agressivas ao meio ambiente.

Nesse contexto, diversos estudos foram e vem sendo desenvolvidos voltados para inibidores naturais, de origem vegetal, como alternativas promissoras por serem provenientes de fontes renováveis, biodegradáveis, de fácil aquisição e baixo custo, que apresentam diferentes compostos orgânicos, a exemplo dos taninos, alcaloides, pigmentos, saponinas, carboidratos, proteínas e aminoácidos, dos quais alguns possuem capacidade inibidora de corrosão $[10,11]$.

A mamona (Ricinus communis) pertence à família botânica Euphorbiaceae, Figura 1a,sendo uma cultura industrial que tem como principal objetivo o óleo (Figura 1b), mas da mamona tudo se aproveita: a torta, as folhas, as hastes e seu principal componente é o ácido ricinoleico, conforme dados de composição apresentados na Tabela 1. A partir da saponificação do óleo da mamona é possível se obter um tensoativo que será estudado no presente trabalho como alternativa sustentável na inibição da corrosão em meio salino.

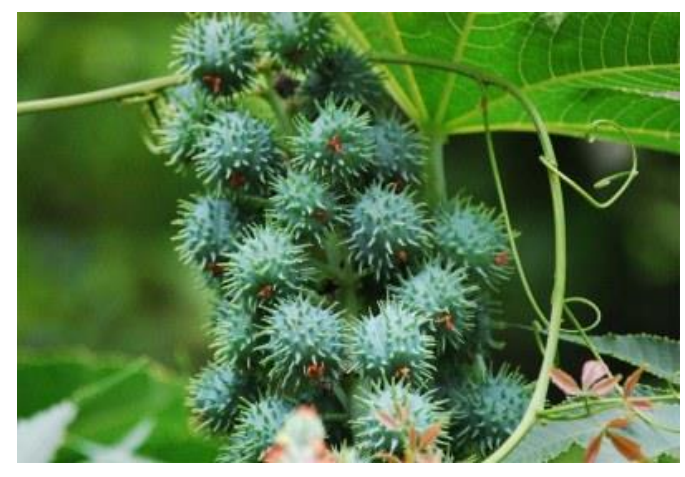

(a)

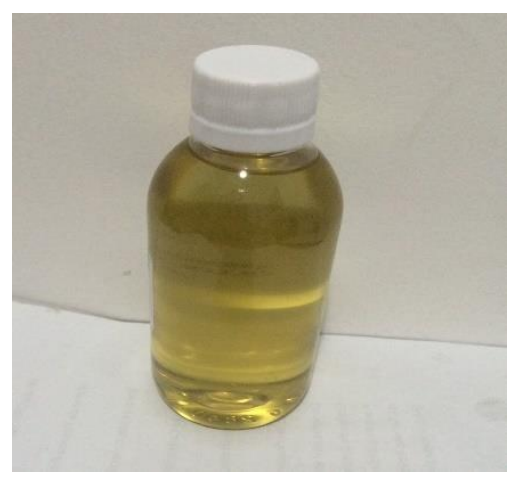

(b)

Figura 1: (a) Exemplar de mamona; (b) óleo de mamona 
Tabela 1: Composição do óleo de mamona

\begin{tabular}{|c|c|c|c|}
\hline $\begin{array}{l}\text { Ácidos } \\
\text { graxos }\end{array}$ & $\begin{array}{l}\text { Número de } \\
\text { carbono }\end{array}$ & Estrutura Química & $\begin{array}{c}\text { Óleo de mamona } \\
(\%)\end{array}$ \\
\hline Esteárico & 18 & & 3 \\
\hline Ricinoléico & 18 & & 86 \\
\hline Oléico & 18 & & 8 \\
\hline Linoléico & 18 & & 3 \\
\hline
\end{tabular}

Óleos vegetais saponificados vêm sendo foco de estudos por serem tensoativos capazes de aderir a superfície metálica formando uma camada protetora.

Os tensoativos, também conhecidos como surfactantes, são moléculas anfifílicas que tem como característica básica possuir em meio aquoso duas regiões de solubilidade diferentes, uma parte polar que possui afinidade pela água (grupo de cabeça - hidrofílico) e outra apolar que possui afinidade por compostos orgânicos (cauda carbônica - hidrofóbico). A Figura 2 apresenta a estrutura básica de um tensoativo.

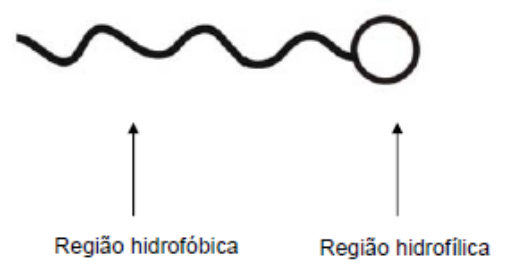

Figura 2: Representação esquemática de uma molécula de tensoativo[12]

Por ser anfifílica, a estrutura molecular de substâncias tensoativas apresentam capacidade de diminuir a tensão interfacial/superficial sendo essa a característica que torna os tensoativos tão importantes na indústria, podendo ser utilizados na estabilização de emulsões e microemulsões, como agentes molhantes, espumantes na superfície de um líquido, detergents, solubilizantes, dentre outros.

Quando as moléculas de um tensoativo são adicionadas a soluções aquosas, as cabeças polares ficam em contato direto com a água enquanto as caudas hidrofóbicas se voltam para o interior, local onde o contato com o diluente seja mínimo. Ocorre uma troca da ligação entre as moléculas de água pelo grupo hidrofílico do tensoativo que possui uma menor força de atração, provocando uma redução na tensão interfacial ou superficial, fator que leva os tensoativos a se adsorverem nas interfaces.

A característica de orientação da molécula é a principal diferença dos tensoativos entre solutos, que tendem a se dissipar por toda solução, a exemplo dos sais orgânicos. Em altas concentrações ocorre a saturação dos tensoativos na interface e eles tendem a se orientar para o seio da solução em forma de monômeros e micelas que se associam espontaneamente em solução aquosa a partir de certa concentração micelar crítica (CMC), formando agregados moleculares de dimensões coloidais. Essas micelas são termodinamicamente estáveis e facilmente reprodutíveis, e não sendo estáticas se formam como agregados [14], ou seja, abaixo da CMC o tensoativo se encontra predominantemente na forma de agregado monomérico, quando pouco acima da CMC existe um equilíbrio dinâmico entre monômeros e micelas, como mostra a Figura 3. 


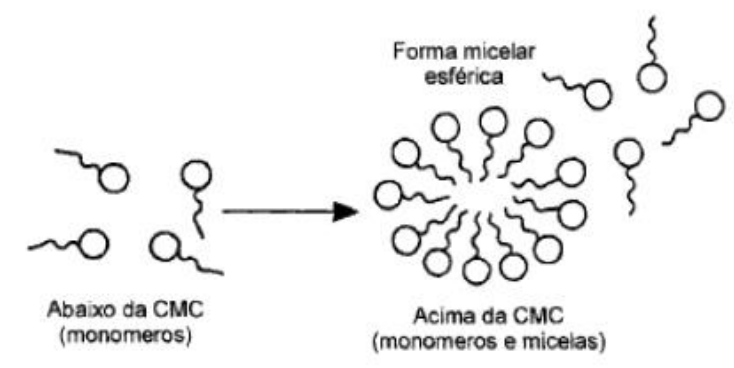

Figura 3: Formação do agregado micelar [17]

Para o presente trabalho, o óleo de mamona foi saponificado para a obtenção de um tensoativo, aqui chamado de óleo de mamona saponificado (OMS), e avaliado quanto à capacidade de inibição da corrosão frente ao aço carbono.

\section{MATERIAL E MÉTODOS}

\subsection{Caracterização do óleo}

O índice de acidez que pode ser definido como o número de miligramas (mg) de hidróxido de sódio $(\mathrm{NaOH})$ necessários para neutralizar os ácidos livres de um grama da amostra.

A análise do índice de acidez revela o estado de conservação dos óleos e gorduras, pois os óleos vegetais ao serem submetidos a diferentes condições como, por exemplo, o contato com altas temperaturas ou outros compostos químicos podem ser degradados modificando sua estrutura e interferindo na qualidade do produto final [16]. Esse índice foi determinado pelo método titulométrico empregando como solução titulante, o $\mathrm{NaOH} 1 \mathrm{M}$ e a fenoftaleína como indicador [17].

O índice de saponificação representa a quantidade de hidróxido de potássio $(\mathrm{KOH})$, em miligramas, capaz de saponificar 1 grama de óleo. A partir do valor desse índice adiciona-se um excesso de $20 \%$ de $\mathrm{KOH}$ para garantir que todo óleo será saponificado transformando-se no tensoativo a ser avaliado no presente trabalho. O método utilizado também foi titulométrico com a fenolftaleína com indicador e a solução de ácido clorídrico (HCl) 5M como titulante [17].

\subsection{Medidas de polarização linear}

Os ensaios foram realizados no potenciostato/galvanostato (PGSTAT 302) à temperatura ambiente, em célula de $250 \mathrm{~mL}$ utilizando volume de solução fixo de $80 \mathrm{~mL}$ e três eletrodos: eletrodo de referência (fio de prata), contraeletrodo (fio de platina) e o eletrodo de trabalho de aço carbono API5LX Gr X42 com área superficial exposta de $1 \mathrm{~cm}^{2}$ (Figura 4).

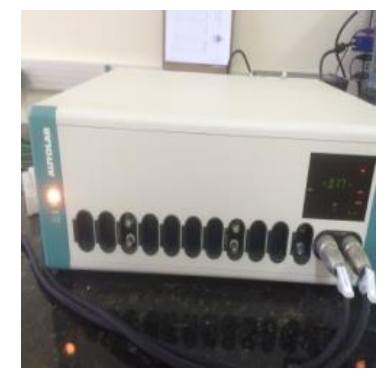

(a)

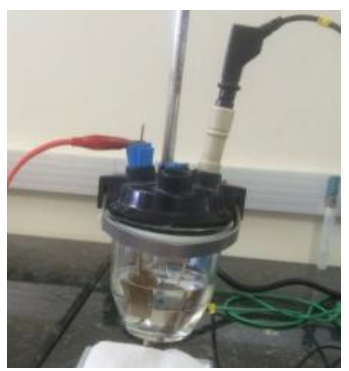

(b)

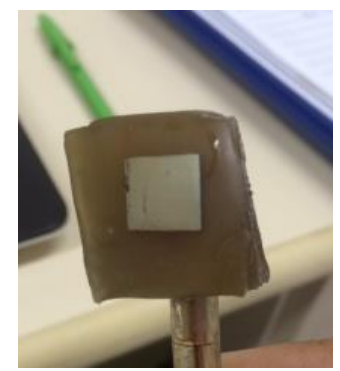

(c)

Figura 4: (a) Potenciostato, (b) célula eletrolítica de 3 eletrodos e (c) eletrodo de trabalho

A eficiência de inibição à corrosão foi avaliada pela técnica de voltametria de varredura linear em solução de $\mathrm{NaCl}$ 3,5\% nas medições em aço carbono. A corrente aplicada ao eletrodo foi 
controlada pelo instrumento (Potenciostato/Galvanostato) possibilitando a medição da diferença de potencial elétrico entre o eletrodo de trabalho e o de referência com velocidade de varredura de 1 $\mathrm{mV} / \mathrm{s}$ e $\pm 300 \mathrm{mV}$ do Potecial de Circuito Aberto, condicionamento de corrente zero em que todas as amostras são submetidas inicialmente.

Os resultados de eficiência de inibição à corrosão, $\mathrm{E} \%$, do aço carbono foram avaliados por dado experimentais dos valores das correntes de corrosão $i$, através da equação 5 .

$$
E \%=\frac{100 \times\left(i_{\text {corr }}-i^{\prime}{ }_{\text {corr }}\right)}{i_{\text {corr }}}
$$

Onde $i_{\text {corr }}$ e $i_{\text {'corr }}$ representam as correntes de corrosão na ausência e presença do inibidor, respectivamente, estimados através do método de extrapolação das curvas de Tafel [7]. Para obtenção dos valores de eficiência foi utilizado o método de extrapolação das curvas de Tafel (obtidas através das curvas de polarização) possibilitando assim a obtenção dos valores das correntes (i) que por sua vez forneceram as variações de inibição. O estudo da eficiência de inibição à corrosão foi realizado a temperatura ambiente, em solução de $\mathrm{NaCl} 3,5 \%$, variando as concentrações do OMS livre. É importante ressaltar que, um inibidor pode ser considerado eficiente quando a corrente elétrica que percorre o sistema é reduzida na presença do inibidor [18].

\section{RESULTADOS E DISCUSSÃO}

\subsection{Caracterização do óleo}

O índice de acidez fornece a quantidade de óleos insaturados no óleo de mamona. O valor obtido para esse índice foi de $0,119 \mathrm{mg} \mathrm{KOH} / \mathrm{g}$. Costa (2006) [19] comenta que valores abaixo de $1 \%$ são considerados comercialmente como tipo 1, de melhor qualidade.

$\mathrm{O}$ índice de saponificação é importante para se obter a quantidade mínima de $\mathrm{KOH}$ necessária para saponificar determinada quantidade de óleo. O valor encontrado para o óleo de mamona em estudo foi de 161,73 mg de $\mathrm{KOH}$ para saponificar 1 grama do óleo. Valor que se encontra próximo a faixa ideal existente na literatura, entre 176-184 mg KOH/g, os valores de índice de saponificação podem ser inferiores devido a menor refinamento do óleo utilizado [20].

\subsection{Medidas de eficiência de inibição à corrosão para o OMS livre}

A Figura 5 apresenta as curvas de polarização de Log da corrente (A) versus Potencial aplicado (V) do branco e do OMS em diferentes concentrações, de $0,1 \mathrm{M}$ à $0,001 \mathrm{M}$. Pode-se verificar que com a adição do inibidor OMS ocorre um deslocamento do potencial de corrosão para a direita, ou seja, valores mais positivos, demonstrando a diminuição do potencial de corrosão que é representado pela interseção das curvas anódicas e catódicas de Tafel, consequentemente reduzindo as reações de corrosão.

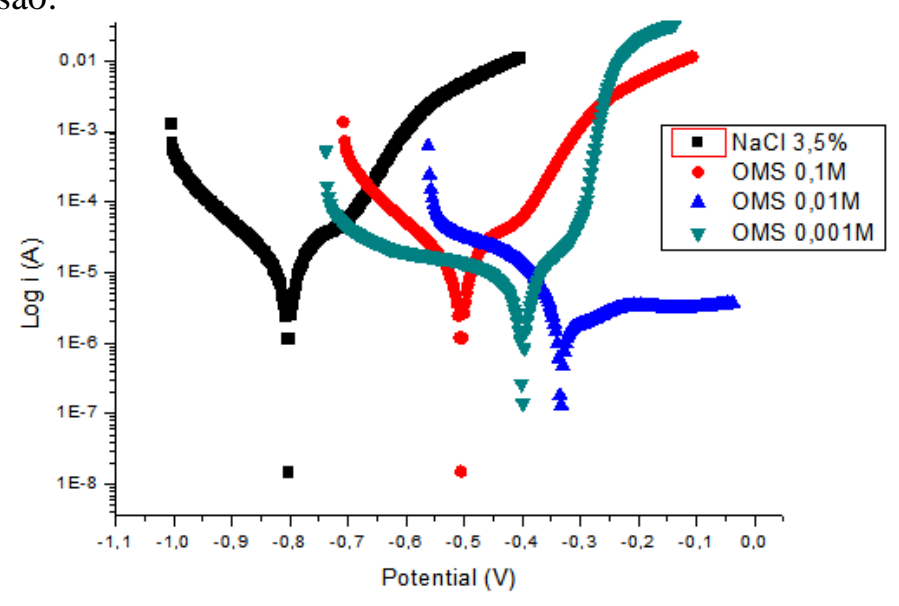

Figura 5: Extrapolação das Curvas de Tafel para o OMS livre 
Na Tabela 2 observa-se os valores dos parâmetros eletroquímicos como coeficientes de Tafel anódicos $\left(b_{a}\right)$ e catódicos $\left(b_{c}\right)$, potencial calculado $\left(\mathrm{E}_{\mathrm{c} 1}\right)$ e observado $\left(\mathrm{E}_{\mathrm{c} 2}\right)$, intensidade da corrente $\left(\mathrm{i}_{\text {corr }}\right)$, taxa de corrosão $(\mathrm{CR})$ e resistência à polarização $\left(\mathrm{R}_{\mathrm{p}}\right)$, obtidos pelo software Nova 1.9.

Tabela 2: Resultados dos parâmetros eletroquímicos obtidos a partir da curva de polarização

\begin{tabular}{rcccc}
\hline Dados & $\begin{array}{c}\text { NaCl } \\
\text { 3,5\% (branco) }\end{array}$ & $\begin{array}{c}\text { OMS livre } \\
\mathbf{0 , 0 0 1 M}\end{array}$ & $\begin{array}{l}\text { OMS livre } \\
\mathbf{0 , 0 1 M}\end{array}$ & $\begin{array}{l}\text { OMS livre } \\
\mathbf{0 , 1 M}\end{array}$ \\
\hline $\mathrm{b}_{\mathrm{a}}(\mathrm{mV} / \mathrm{dec})$ & 114,00 & 118,020 & 43,668 & 35,576 \\
$\mathrm{~b}_{\mathrm{c}}(\mathrm{mV} / \mathrm{dec})$ & 78,865 & 71,085 & 113,130 & 41,934 \\
$\mathrm{E}_{\mathrm{c} 1}(\mathrm{mV})$ & $-804,890$ & $-399,730$ & $-333,090$ & $-452,28$ \\
$\mathrm{E}_{\mathrm{c} 2}(\mathrm{mV})$ & $-804,320$ & $-399,560$ & $-333,510$ & $-451,92$ \\
$\mathrm{i}_{\mathrm{corr}}(\mu A)$ & 10,350 & 4,622 & 1,924 & 1,073 \\
$\mathrm{CR}(\mathrm{mm} / \mathrm{y})$ & 0,1202 & 0,0544 & 0,0223 & 0,0125 \\
$\mathrm{R}_{\mathrm{p}}(k \Omega)$ & 1,956 & 4,114 & 7,111 & 7,788 \\
\hline
\end{tabular}

Observa-se que os resultados dos potenciais de corrosão calculados $\left(\mathrm{E}_{\mathrm{cl}}\right)$, são bem semelhantes ao potencial de corrosão observado $\left(\mathrm{E}_{\mathrm{c} 2}\right)$, validando assim os experimentos. Pode-se verificar também que os dados da intensidade de corrente diminuem à medida que se aumenta a concentração de tensoativo, ou seja, ocorre um decréscimo da intensidade das reações de corrosão, confirmando assim a inibição devido a camada protetora do tensoativo sobre o metal. Os valores dos coeficientes anódicos $\left(b_{a}\right)$ e catódicos $\left(b_{c}\right)$ variam para as diferentes concentrações de OMS indicando que o inibidor é capaz de controlar tanto as reações de corrosão anódicas quanto catódicas, ou seja, o filme protetor pode ser formado antes de ambas as reações. A resistência a polarização $\left(R_{p}\right)$ representa a resistência do metal à corrosão devido a corrente aplicada e pelos valores obtidos podese observar que ocorre aumento da $\mathrm{R}_{\mathrm{p}}$ com aumento da concentração do tensoativo que é gerado pelo efeito de inibição do filme adsortivo formado [21].

$\mathrm{Na}$ Tabela 3 é possível observar os resultados de eficiência da inibição da corrosão.

Tabela 3: Valores de eficiência de inibição da corrosão para o OMS

\begin{tabular}{cccc}
\hline \multirow{2}{*}{ Eficiência } & OMS & OMS & OMS \\
& $\mathbf{0 , 0 0 1 M}$ & $\mathbf{0 , 0 1 M}$ & $\mathbf{0 , 1 M}$ \\
\hline \multirow{2}{*}{ E\% (i corr $)$} & 55,3 & 81,4 & 89,6 \\
\hline
\end{tabular}

É possível observar que o OMS é capaz de inibir a corrosão e que a sua eficiência aumenta à medida que a concentração do OMS aumenta. Isso ocorre pois acima da concentração micelar crítica, que Moura et al. (2009) [22] apresenta para o OMS na faixa do $10^{-2} \mathrm{M}$, as moléculas formam um filme micelar em toda extensão da superfície do metal, melhorando a adesão e proteção se comparado a concentração inferior de $10^{-3} \mathrm{M}$ onde existe apenas a presença dos monômeros do tensoativo.

\section{CONCLUSÃO}

O resultado obtido para o índice de acidez de $0,119 \mathrm{mg} \mathrm{KOH} / \mathrm{g}$ demonstra que o óleo utilizado é do tipo 1, por ser inferior a 1\% [19], garantindo assim a qualidade do óleo de mamona e o índice de saponificação obtido de $161,73 \mathrm{mg} \mathrm{KOH} / \mathrm{g}$ de óleo foi considerado satisfatório apesar de estar um pouco abaixo dos valores encontrados na literatura [20].

Os resultados de eficiência obtidos pelas análises de polarização linear comprovaram a ação inibitória do óleo de mamona saponificado à corrosão do aço em meio salino uma vez que ocorreu decréscimo da corrente de corrosão e deslocamento do potencial de corrosão para valores mais positivos, obtendo uma eficiência de 89,6\% para uma concentração de OMS de 0,1 M. 


\section{AGRADECIMENTOS}

Os autores agradecem ao Programa de Pós-Graduação em Engenharia Química da Universidade Federal de Sergipe (PEQ/UFS) pela oportunidade de realizar o estudo apresentado neste trabalho e à Universidade Federal do Rio Grande do Norte (UFRN) pela disponibilização do laboratório para realização dos experimentos de polarização linear.

\section{REFERÊNCIAS BIBLIOGRÁFICAS}

1. Thomas JE. Fundamentos de Engenharia de Petróleo. Rio de Janeiro: Interciência; 2004. 272p.

2. Gomes EA.Tratamento combinado de água produzida de petróleo por eletroflotação e processo fenton. [Dissertação de Mestrado em Engenharia de Processos]. Aracaju (SE): Universidade Tiradentes em Aracaju; 2009. 84p.

3. Stewart M, Arnold K. Produced Water Treatment Field Manual. Amsterdam:Gulf Professional Publishing; 2011. 244p.

4. Almeida CC. Avaliação de inibidores verdes microemulsionados na inibição à corrosão do aço carbono AISI 1020. [Dissertação de Mestrado em Engenharia de Materiais]. Natal (RN): Universidade Federal do Rio Grande do Norte em Natal; 2012. 85p.

5. Moreira RR. Estudo da corrosão e inibição dos aços carbono AISI 1010 e inoxidável AISI 316 e duplex UNS S31803 em meio de solução de íons cloreto. [Dissertação de Mestrado em Química]. Vitória (ES): Universidade Federal do Espírito Santo em Vitória; 2014. 123p.

6. Gentil V. Corrosão. Rio de Janeiro: LTC; 2011. 332p.

7. Wolynec S. Técnicas Eletroquímicas em Corrosão. São Paulo: USP; 2003. 166p.

8. Jambo HCM, Fofano S. Corrosão-Fundamentos, Monitoração e Controle. Rio de Janeiro: Ciência Moderna Ltda; 2008. 342p.

9. Roberto EC. Caracterização e aplicação de sistemas micelares e micromeulsionados como inibidores de corrosão. [Dissertação de Mestrado em Química]. Natal (RN): Universidade Federal do Rio Grande do Norte em Natal; 2010. 150p.

10. Alencar MFA, Oliveira LRF, Gomes RF, Gomes FFS, Araújo Neto JAM, Ferreira Júnior JM, Silva RCB. Extratos de plantas da Caatinga como inibidor de corrosão. $5^{\circ}$ Congresso Norte-Nordeste de Química e $3^{\circ}$ Encontro Norte-Nordeste de Ensino de Química. Natal (RN). 2013.

11. Felipe MBMC, Maciel MAM, Medeiros SRB, Silva DR. Aspectos Gerais Sobre Corrosão e Inibidores Vegetais. Rev Virt de Quim. 2013;5:746-758.

12. Rodrigues CJF. Estudo da eficiência do tensoativo sorbitado tween 80 veiculado em nanoemulsão contendo óleo de soja, como inibidor de corrosão. [Dissertação de Mestrado em Química]. Natal (RN): Universidade Federal do Rio Grande do Norte em Natal; 2012. 107p.

13. Moura JIP. Efeitos de um inibidor comercial na inibição à corrosão do aço API5LX GR X42 em meio de cloreto e oxigênio. [Dissertação em Mestrado em Engenharia Química]. Natal (RN): Universidade Federal do Rio Grande do Norte em Natal; 2006. 126p.

14. Maniasso N. Ambientes micelares em química analítica. Quim Nova. 2001;24:87-93.

15. Moreto E, Fett R. Óleos e gorduras vegetais: processamento e análises. Florianópolis: UFSC; 1989. 179p.

16. Instituto Adolfo Lutz. Normas analíticas, métodos químicose físicos para analisesde alimentos. São Paulo: Instituto Adolfo Lutz; 2008. 1020p.

17. El-Achouri M, Hajji MS, Salem M, Kertit S, Aride J, Coudert R, Essassi EM. Some Nonionic Surfactants as Inhibitors of the Corrosion of Iron in Acid Chloride Solutions. Corrosion Sci. 1996;52:103-108, doi:10.5006/1.3292100.

18. Costa TL, Duarte, MEM, Beltrão NE, Severino LV, Paixão FJR, Ribeiro D. Estudo da viscosidade do óleo de mamona para temperaturas na faixa de 20 a $80^{\circ} \mathrm{C}$. II Congresso Brasileiro de Plantas Oleaginosas, Óleos, Gorduras e Biodiesel. Varginha (MG); 2005

19. Costa TL. Características físicas e físico-químicas do óleo de duas cultivares de mamona. [Dissertação de Mestrado em Engenharia Agrícola]. Campina Grande (PB): Universidade Federal de Campina Grande; 2006. 113p.

20. Costa HM, Ramos VD, Abrantes TASA., Castro LLY, Visconte LLY, Nunes RCR, Furtado CRG. Polímeros: Ciência e Tecnologia. 2004;14:46-50. doi:http://dx.doi.org/10.1590/S010414282004000100013 
21. Al-Sabagh AM, Nasser NM, Khamis EA, Mahmoud T. Synthesis of non-ionic surfactants based on alkylene diamine and evaluation of their corrosion inhibition efficiency on carbono steel in formation water. Egyptian Journal of Petroleum 2017;26:41-51, doi:10.1016/j.ejpe.2016.03.001

22. Moura EF, Wanderley Neto AO, Dantas TNC, Scatena Júnior H, Gurgel A. Applications of micelle and microemulsion systems containing aminated surfactants synthesized from ricinoleic acid as carbon-steel corrosion inhibitors. Colloids and Surfaces. A, Physicochemical and Engineering Aspects. 2009;340:199207, doi:10.1016/j.colsurfa.2009.03.031 